\title{
Continuidades y rupturas de la protesta universitaria en el Chile de la posdictadura (1990-2014) ${ }^{1}$
}

\author{
Cristóbal Alejandro Villalobos Dintrans ${ }^{2}$ \\ Camila Ortiz Inostroza ${ }^{3}$
}

Fecha de recepción: 20 de marzo de 2019

Fecha de aprobación: 6 de mayo de 2019

Fecha de publicación: 30 de julio de 2019

\begin{abstract}
Resumen
El artículo busca analizar históricamente los ciclos, tendencias y trayectorias de conflictividad universitaria en el Chile posdictadura (1990-2014). Mediante una investigación descriptiva y cuantitativa, que utiliza como técnica de recolección de datos el Análisis de Eventos de Protesta (AEP), el estudio analiza tres aspectos centrales de la trayectoria de las protestas estudiantiles: la cantidad de protestas desarrolladas en el periodo; las demandas generadas y tácticas utilizadas durante estos procesos, y la intensidad de estas acciones. Los resultados permiten matizar, complementar y disputar algunas de las principales tesis esgrimidas por la literatura hasta la fecha, ya que muestran i) que las protestas universitarias se han desarrollado a través de un patrón de comportamiento cíclico durante todo el periodo, intercalándose momentos de alza y baja en la conflictividad, ii) un proceso de heterogenización de las demandas y un aumento de las marchas como táctica prototípica de acción, y iii) un incremento en la cantidad de participantes y ampliación de la extensión territorial de los eventos durante la última década, pero no de la duración de las acciones de protestas.
\end{abstract}

Palabras clave: protesta, movimiento estudiantil, universitarios, Chile, posdictadura

El presente artículo procede de la tesis de investigación doctoral de Cristóbal Villalobos titulada: "Los conflictos sociales en el campo educativo en el Chile postdictadura (1990-2014). Análisis de su evolución, principales características y factores relacionados"; y de la tesis de Magíster de Camila Ortiz titulada: "Estructura social y movimiento estudiantil en el Chile post-dictadura (1990-2014)". Los autores agradecen el apoyo brindado por el Centro de Conflictos y Cohesión Social (CONICYT-FONDAP 15130009) y al Programa de Becas Nacionales de Magíster y Doctorado de CONICYT. 2 Doctor en Ciencias Sociales, Universidad de Chile. Investigador Asociado, Centro de Políticas y Prácticas en Educación (CEPPE UC), Pontificia Universidad Católica de Chile, Chile. Contacto: clvillal@uc.cl

3 Magíster en Ciencias Sociales, Universidad de Chile. Estudiante de Doctorado en Sociología, Pontificia Universidad Católica de Chile, Chile. Contacto: cfortiz3@uc.cl 


\title{
Continuities and ruptures of university protest in Chilean post-dictatorship (1990-2014)
}

\begin{abstract}
The article analyzes the historical cycles, trends and trajectories of university conflict in post-dictatorship Chile (1990 - 2014). Through a descriptive and quantitative research study, which uses the Protest Event Analysis (PEA) as a data collection technique, the study analyzes three central aspects of the trajectory of student protests: the number of protests developed during postdictatorship, the demands and tactics used during these processes, and the intensity of these actions. The delivered results help to explain, complement and dispute the main theses generated by academic literature. The results show: i) that the protests have developed a cyclical nature over the period, with periods of rise and fall during the 25 years under analysis; ii) a process of heterogenization of demands and an increase in marches as a prototypical tactic; iii) an increase in the number of participants during the last decade, though no rise in the duration of protest.
\end{abstract}

Keywords: protest, student movement, university students, Chile, postdictatorship

\section{Continuidades e rupturas dos protestos universitários no Chile da pós-ditadura (1990-2014)}

\begin{abstract}
Resumo
$\mathrm{O}$ artigo busca analisar historicamente os ciclos, tendências e trajetórias de conflitividade universitária no Chile pós-ditadura (1990-2014). Através de uma pesquisa descritiva e quantitativa, que utiliza como técnica de coleta de dados a Análise de Eventos de Protesto (AEP), o estudo analisa três aspectos centrais da trajetória dos protestos estudantis: a quantidade de protestos desenvolvidos no período; as demandas geradas e tácticas utilizadas durante estes processos, e a intensidade destas ações. Os resultados permitem matizar, complementar e disputar algumas das principais teses apresentadas pela literatura até a data, já que mostram i) que os protestos universitários têm se desenvolvido através de um padrão de comportamento cíclico durante todo o período, intercalando-se momentos de subida ou descida na conflitividade, ii) um processo de heterogeneização das demandas e um aumento das marchas como táctica prototípica de ação, e iii) um incremento na quantidade de participantes e ampliação da extensão territorial dos eventos durante a última década, mas não da duração das ações de protestos.
\end{abstract}

Palavras-chave: protestos, movimento estudantil, universitários, Chile, pósditadura 


\section{Introducción}

Actualmente, el análisis de los movimientos sociales y procesos de conflictividad constituye un corpus académico de nivel mundial. A diferencia de los estudios desarrollados en los sesenta y setenta, que buscaron generar investigaciones teóricoempíricas que buscaban sintetizar funcionalismo y marxismo, y que tuvieron como principales exponentes a Ralph Dahrendorf, Randall Collins y Charles Wrigth Mills, la nueva oleada académica de estudios sobre conflictos se ha desarrollado desde múltiples perspectivas conceptuales, utilizando variadas miradas metodológicas y analizando casos de todo el mundo (Della Porta, Kriesi y Rutch, 2009; Snow, Soule y Kriesi, 2007), constituyendo un campo de investigación cada vez más denso y complejo.

Uno de los objetos más estudiados por este campo han sido los movimientos universitarios. Aunque existe evidencia de la importancia de estos conflictos en la construcción de los sistemas educativos desde el siglo XIX (Petitat, 1987), desde finales de los años sesenta del siglo XX estos movimientos son entendidos como uno de los mayores representantes de los nuevos tipos de movilización social, especialmente en los países desarrollados (Touraine, 1970). El movimiento estudiantil francés de mayo de 1968, la movilización de estudiantes postsecundarios en 1968 y 1969 en Praga, o los movimientos universitarios en contra de la guerra de Vietnam en Estados Unidos son ejemplos clásicos de este punto de quiebre (Seferiades, 2010).

En Chile, el movimiento de reforma universitaria de finales de los sesenta — que generó importantes cambios en los procesos de acceso, gobernanza y organización de las universidades- constituye un hito que marca un antes y un después en los estudios sobre movimientos estudiantiles (Brunner y Flisfisch, 2014), motivando nuevas investigaciones académicas sobre el rol de la universidad y de los estudiantes en la sociedad (Reca, 1970; Vasconi, 1968). Este impulso, sin embargo, se verá 
eclipsado por el golpe militar, que hará dirigir la discusión intelectual a otros sujetos sociales (especialmente los pobladores y los trabajadores) o a otras temáticas (como el rol de la democracia o las reformas sociales y económicas implementadas por la dictadura). Este relativamente bajo interés académico por los movimientos estudiantiles universitarios se mantendrá posteriormente durante gran parte de los años noventa.

Sin embargo, desde comienzos del milenio la discusión sobre las características, formas de organización y/o lógicas de operación de los movimientos universitarios en Chile ha cobrado un gran y explosivo interés, motivado por los procesos de movilización generados durante la última década (Aguilera y Álvarez, 2015; Montero, 2018). Una reciente revisión sistemática de los estudios sobre el movimiento universitario chileno, publicados entre 2000 y 2016 (Asún, Zúñiga, Yañez y Villalobos, 2018) ha encontrado que, durante este periodo, se han publicado un total de 149 investigaciones académicas, incluyendo principalmente ensayos demostrativos $(37,7 \%)$, estudios empíricos $(34,2 \%)$ y ensayos teóricos $(16,1 \%)$. Vistas en su conjunto, estas investigaciones comparten dos características centrales. Por una parte, la mayoría se ha desarrollado utilizando técnicas de investigación cualitativa o mediante ensayos interpretativos, siendo poco frecuentes las investigaciones cuantitativas o con fuentes de datos secundarios. Por otra parte, casi todos los estudios han puesto el foco específico en un año particular (especialmente en 2006 y 2011), siendo escasas las investigaciones con foco en el análisis histórico del movimiento estudiantil. Considerando lo anterior, el presente artículo busca aportar al estudio del movimiento universitario chileno buscando llenar estos vacíos investigativos. De esta manera, y a través de un estudio que tiene como objetivo fundamental examinar históricamente los ciclos, tendencias y trayectorias de conflictividad universitaria entre 1990 y 2014, se busca analizar las continuidades y rupturas del movimiento estudiantil en el Chile posdictadura, alimentando así el campo de investigación existente. 
Para llevar adelante este objetivo, el artículo se estructura en cinco apartados, incluida esta introducción. En el segundo apartado se describen los antecedentes conceptuales del estudio, enfocados en tres aspectos: definir las categorías de acción colectiva, protestas y movimientos sociales; dar cuenta de la literatura existente para analizar las trayectorias, tendencias y ciclos de conflictividad, y describir las principales interpretaciones y tesis utilizadas en estudios previos para explicar los procesos de conflictividad en el campo universitario en el Chile posdictadura. El tercer apartado detalla los aspectos metodológicos del estudio, dando cuenta de la forma de levantamiento y extracción de datos, y de los mecanismos de análisis de los mismos. En el cuarto apartado se reportan los resultados de investigación, resaltando principalmente tres aspectos: i) la trayectoria histórica de la conflictividad universitaria; ii) la evolución de las principales características de la conflictividad universitaria (duración y participantes); y iii) las continuidades y rupturas de las demandas desplegadas por estos actores. Finalmente, el quinto apartado busca sintetizar las principales conclusiones del estudio, discutiendo especialmente sobre la trayectoria de la conflictividad estudiantil en el Chile posdictadura.

\section{Marco conceptual}

Para estudiar la trayectoria histórica de la conflictividad universitaria en Chile entre 1990 y 2014 es necesario comprender los fundamentos conceptuales desarrollados en las últimas décadas sobre los ciclos de protestas. Utilizando como referencias principales los aportes teóricos de Tarrow y Tilly, el siguiente apartado define en primer término el objeto central de la investigación: los conflictos o protestas sociales. A partir de este punto se exponen los conceptos usados para analizar históricamente los conflictos y protestas, destacando especialmente las nociones de "ciclos", "olas" y "peaks", para posteriormente finalizar con la descripción de las principales tesis esbozadas respecto de la dinámica y 
trayectoria de conflictividad en el caso del movimiento universitario chileno en la posdictadura.

\section{i) Acción colectiva, protesta y movimiento social}

Dentro del espectro de conductas y fenómenos que pueden ser englobados en la categoría de "acción colectiva", es reconocida la distinción entre acción colectiva institucionalizada y no institucionalizada. Sintéticamente, esta distinción busca dar cuenta de, por un lado, formas de acción que se producen en el marco de las instituciones políticas y sociales, generalmente por parte de grupos constituidos que operan en nombre de ciertos objetivos comunes; en contraste con aquellas formas de acción que tienen cabida por fuera de los marcos y procedimientos de las instituciones políticas (Tarrow, 1997; Von Bülow y Donoso, 2017; Treviño, Villalobos, Béjares y Naranjo, 2018). Dentro de esta segunda categoría, es decir, de las formas de acción colectiva no institucionalizadas, es posible encontrar una variada gama de acciones, incluyendo manifestaciones callejeras, firma de peticiones, boicots al consumo, tomas de espacios públicos o privados, boicots o huelgas, actos poéticos o teatrales, entre otras, que, vistas en su conjunto, pueden ser definidas como protestas o procesos de conflictividad social.

Conceptualmente, las protestas o conflictos tienen tres características básicas. Por una parte, todas las protestas tienen un carácter contencioso, ya que tienen como objetivo fundamental la interrupción, obstrucción o generación de incertidumbre sobre las actividades de otros, buscando así subvertir el espacio o tiempo rutinario de una sociedad (Tarrow, 1997). En segundo término, las protestas o conflictos son acciones públicas y colectivas, lo que les confiere su carácter social. De esta manera, las protestas son acciones que tienen un objetivo público de transformación, aun cuando algunas veces se desarrollen al interior de espacios privados, como ocurre con las huelgas o las tomas (Della Porta, 2008). Finalmente, las protestas buscan dar cuenta de una dife- 
rencia o disputa al interior de un Estado sin la utilización (masiva) de armas. En consecuencia, las protestas excluyen disputas como la guerra de guerrillas, los conflictos armados o los genocidios, diferenciándose de la noción de conflicto utilizada por Calderón (2013). Entonces, las protestas o conflictos pueden entenderse como acciones públicas y colectivas, desarrolladas por uno o más actores, que buscan dar cuenta de una contradicción o diferencia en alguna temática al interior de un Estado, incluyendo acciones de confrontación, acciones violentas no armadas y acciones culturales.

Las protestas constituyen parte fundamental de la conformación de los movimientos sociales, aunque ambos conceptos no son equiparables, por tres razones. En primer lugar, los movimientos sociales son formas de acción colectiva que, aunque desarrollan protestas, también utilizan otros tipos de participación política, como la negociación, el lobby o las alianzas políticas (Tarrow, 1997). En segundo lugar, los movimientos sociales se dirigen hacia oponentes mediante secuencias de interacción mantenidas en el tiempo, que incluyen momentos de protestas y momentos de organización (Tilly y Wood, 2013), siendo, por lo tanto, las protestas solo una reducida parte del marco de acción de los movimientos sociales. Por lo mismo, estudiar protestas puede implicar desconocer ciertos aspectos de los movimientos. Finalmente, los movimientos sociales requieren, para su constitución, del desarrollo de capacidades y recursos para coordinar y mantener la interacción entre movilizados y oponentes dentro de un determinado periodo, aspectos que no son necesariamente visibles al analizar exclusivamente las protestas.

\section{ii) El análisis de la trayectoria de las protestas y los movimientos sociales}

Como mencionamos, una de las características centrales de los movimientos sociales es su dimensión temporal. Una expresión de ello dice relación con la dinámica recurrente a través de 
la cual se desenvuelven las protestas de estos movimientos. Dos grandes hallazgos se han realizado respecto de este tópico.

En primer término, y a partir de un estudio pionero, Koopmans (1993) identificó que los movimientos sociales tienden a desarrollarse a través de olas o ciclos de protesta, en los que se intercalan periodos de incremento con periodos de baja conflictividad. Mientras en los momentos álgidos de conflictividad la protesta se difunde sectorial y geográficamente, crece la participación, se fortalecen los marcos de sentido compartidos y aumenta considerablemente la interacción entre movilizados y oponentes; en los periodos de declive o estabilización de la conflictividad (de duración variable y que pueden a su vez conducir al inicio de un nuevo momento de auge de la actividad de protesta), en cambio, se produce un debilitamiento de los movimientos sociales y una reestructuración de sus demandas (Tarrow, 1997; Demirel-Pegg, 2014).

En segundo término, y complementando la idea de ciclos u olas de protesta, algunos investigadores han destacado la existencia de cimas o picos (peaks) en los procesos de protesta social desarrollados por los movimientos sociales, los que pueden ser provocados por la propia dinámica del ciclo de protesta, o bien gatillados por eventos puntuales (como por ejemplo una muerte o una sentencia judicial inesperada) que desatan y transforman la trayectoria de los procesos de conflictividad (Koopmans y Rutch, 1999). Más allá del origen de los peaks, lo central es que estos puntos álgidos de protesta son eventos críticos que cambiarían la tendencia de la evolución o trayectoria de la protesta, generando discontinuidades y rupturas no "planificadas" (Salinas, 2016).

Recientemente, múltiples estudios han buscado analizar los movimientos de protesta desde una perspectiva procesual e histórica (Almeida, 2012; Dodson, 2015; Tarrow, 1997). Vistas en su conjunto, estas investigaciones han reconocido que durante las últimas décadas se ha producido un aumento de las 
protestas, junto con un incremento de su protagonismo en la agenda y el debate público, perfilándose de este modo lo que parece ser un ciclo de intensificación global de la protesta social y de masificación de esta forma de acción colectiva, lo que ha llevado a algunos a hablar de la existencia de un "mundo de protestas" (Della Porta, 2008) o de una "sociedad movilizada" (Tarrow, 1997).

\section{c) La protesta universitaria en el Chile posdictadura. Principales tesis}

Como se ha indicado, el movimiento universitario chileno ha cobrado gran interés mediático y académico en la última década. De este conjunto de investigaciones es posible extraer una serie de argumentos a contrastar con la evidencia levantada en esta investigación. La presente sección expone tres de las principales tesis generadas hasta la fecha en este campo de estudio.

Tesis 1: La trayectoria histórica del movimiento universitario puede caracterizarse por un periodo de desmovilización durante la década de 1990 y un aumento de la conflictividad desde los 2000.

A pesar de que el retorno a la democracia en 1990 restituye libertades y genera condiciones para la reorganización de la sociedad civil, diversos autores han descrito el periodo inmediatamente posterior a la transición a la democracia como una época de desmovilización y apatía (Fuentes, 1999; De la Maza, 1999), que en el campo estudiantil se habría expresado en el debilitamiento de las orgánicas estudiantiles y el desprestigio de las juventudes políticas (Thielemann, 2016), contribuyendo de este modo a limitar e inhibir su capacidad de acción. En contraste, durante los años 2000 y particularmente a partir de los acontecimientos del año 2011, se reconocería la irrupción de un nuevo ciclo de conflictividad, logrando romper con la tendencia de escasa movilización que habría caracterizado al campo en el década anterior, generándose así un cambio paradigmático en la trayectoria del movimiento estudiantil universitario (Aguilera y Álvarez, 
2015; Ruiz y Boccardo, 2015; Disi, 2018; Somma, 2012; Salinas y Fraser, 2012; Bellei, Cabalín y Orellana, 2014).

Tesis 2: La trayectoria de la conflictividad universitaria no sólo se caracteriza por un aumento de los niveles de protesta en la última década, sino también por cambios en las demandas (orientación hacia demandas estructurales) y en las acciones (innovaciones tácticas) del movimiento.

Una segunda tesis dice relación con la evolución que habría experimentado el movimiento estudiantil durante las últimas décadas en términos de sus objetivos (demandas) y formas de expresión o repertorios de acción (tácticas). Respecto de los objetivos, la idea central es que el campo habría mutado desde demandas centradas en el financiamiento y el mejoramiento de la calidad educativa a un cuestionamiento global al modelo de mercado que rige al sistema de educación superior (Bellei, Cabalín y Orellana, 2014; Somma, 2012; Espinoza y González, 2014), apelando a transformaciones de corte estructural al sistema (Salinas y Fraser, 2012). Respecto de las tácticas, algunos autores han destacado la centralidad de la marcha como táctica del movimiento (Guzmán-Concha, 2012), mientras otros han relevado el aumento y protagonismo en la última década de un conjunto de repertorios de acción, que incluye desde la ocupación de establecimientos educacionales a las protestas performáticas y/o culturales (Cabalín, 2012; Cabalín y Orellana, 2014; Paredes, Ortiz y Araya, 2018; Ponce y Miranda, 2016; Salinas y Fraser, 2012).

Tesis 3: La trayectoria histórica de la conflictividad universitaria durante la posdictadura se caracterizaría por un aumento de la intensidad de la protesta estudiantil en la última década.

Aunque la dimensión de la intensidad de la movilización estudiantil no ha sido estudiada ni interpretada en la misma medida que la frecuencia, demandas y tácticas de los eventos de protesta, algunos autores han indicado que existirían señales para afirmar que se han generado aumentos en la magnitud de la protesta en los últimos años, expresados en el incremento del 
número de participantes y en la cobertura geográfica e institucional cada vez más amplia de las convocatorias. Algunos han señalado que este fenómeno sería consecuencia principalmente de factores políticos, como la baja legitimidad de las instituciones o el ascenso de la derecha (Disi, 2018; Sherman, Arriagada y Valenzuela, 2015), mientras que otros han puesto el foco en la baja capacidad de gestión del conflicto por parte de las autoridades y en el tipo de estrategias escogidas por las contrapartes (diálogo, tácticas represivas, entre otras) para responder a la interpelación del movimiento en la última década, especialmente visible desde el año 2011 (Guzmán-Concha, 2012). Los factores anteriores, junto con la facilitación de la coordinación y las comunicaciones que proveen las tecnologías digitales, especialmente las redes sociales (Akaev et al., 2017; Bellei, Cabalín y Orellana, 2014), serían las variables más relevantes para explicar la masividad alcanzada por las convocatorias en la última década, que constituiría uno de los rasgos más llamativos de las movilizaciones del periodo post 2000 .

\section{Metodología}

En términos metodológicos, el estudio puede caracterizarse como una investigación descriptiva y cuantitativa. Es descriptiva, pues su objetivo fundamental es dar cuenta de las trayectorias y ciclos de conflictividad del movimiento universitario en el Chile posdictadura, sin pretensiones explicativas respecto de las causas que producen estos procesos, ni de interpretación sobre cómo son vivenciados estos fenómenos por los actores sociales. Es cuantitativa, pues las técnicas de recolección de información y los métodos de análisis buscan segmentar y cuantificar la realidad social (Canales, 2006), estableciendo criterios numéricos para cualificar los procesos de conflictividad.

Considerando lo anterior, tres aspectos metodológicos se describen en este apartado: i) la técnica de recolección de información usada; ii) las características de las fuentes utilizadas y del 
proceso de producción de datos; y iii) las técnicas de análisis utilizadas.

\section{a) Técnica de producción de datos}

El Análisis de Eventos de Protesta (AEP) es una técnica de producción de datos que permite registrar sistemáticamente eventos de conflictividad. Desarrollada desde hace más de 40 años para estudiar procesos de acción colectiva, protestas y conflictividad, el AEP usa una cantidad limitada de fuentes secundarias de información (principal pero no únicamente, archivos de prensa) para procesar y codificar datos de forma estandarizada, permitiendo estudiar históricamente trayectorias y ciclos de movilización y conflictos sociales (Earl, Martin, McCarthy y Soule, 2004; Koopmans y Rutch, 1999), siendo uno de los métodos más utilizados para estudiar fenómenos de conflictividad de mediano y largo plazo (Olzak, 1989).

En términos epistemológicos, los estudios que utilizan el Análisis de Eventos de Protesta comparten dos características. En primer lugar, y al centrar el foco investigativo en fuentes secundarias y no en los actores sociales (Koopmans y Rutch, 1999), se alejan de las perspectivas identitarias, partiendo del supuesto de que es posible captar la dinámica de los conflictos y movimientos sociales a partir de fuentes externas. En segundo término, los análisis realizados con el AEP pueden estudiar de manera privilegiada la interacción entre agencia y estructura, ya que entienden que "el conflicto se compone por unidades particulares - llamadas actos de protesta- pero que se encuentran limitados a una estructura determinada, dentro de las que se desarrollan los procesos de conflictividad" (Villalobos, 2015, p. 60). De esta manera, el Análisis de Eventos de Protesta permite entender históricamente las relaciones e interacción entre los procesos de movilización y las estructuras en que estos se desenvuelven, dando cuenta de sus continuidades y rupturas. 


\section{b) Levantamiento y codificación de datos}

Para analizar la trayectorias y ciclos de la protesta estudiantil mediante el Análisis de Eventos de Protesta se siguieron los cuatro pasos establecidos por la literatura: i) generación de la unidad de análisis; ii) selección de fuentes; iii) generación de protocolo de codificación de datos; y iv) registro y codificación de variables de interés (Koopmans y Rutch, 1999).

Respecto de la unidad de análisis, se definió como objeto central de estudio los conflictos universitarios, entendidos como todos aquellos eventos sociales irruptivos desarrollados en el espacio público, convocados, protagonizados o con presencia de estudiantes universitarios y en los que hayan participado dos o más personas, en línea con el estudio de Olzak y West (1991). En segundo lugar, y respecto de las fuentes seleccionadas, se utilizaron tres medios de prensa nacional: i) El Mercurio; ii) La Tercera; y iii) La Nación. La selección de estas fuentes buscó incluir medios que: i) tuvieran altos volúmenes de información (tiraje diario, alto número de diarios, alcance nacional), lo que permite (teóricamente) ampliar el espectro de extracción de eventos (Koopmans, 2007); ii) medios de prensa que representaran distintas líneas editoriales, a fin de controlar este potencial sesgo (Ortiz, Myers, Walls y Díaz, 2005); y iii) medios de prensa que tuvieran una permanencia en todo el tiempo de estudio (1990-2014), lo que permite dar continuidad temporal al sesgo producido por el medio de prensa seleccionado 4 . Aunque estos criterios no aseguran eliminar la existencia de sesgos (de información o descripción) de las fuentes, sí permiten controlar (especialmente en términos temporales) el efecto de estos sesgos (Río, 2008).

Evidentemente, esto no elimina todos los posibles sesgos de información, considerando la estructura de los medios de prensa del país. Por ello, es relevante destacar la inclusión de un medio estatal (La Nación) junto a los dos mayores medios privados del país (El Mercurio y La Tercera), obteniendo así una especie de "contrabalance" en términos de información y descripción de las noticias. 
Una vez seleccionadas las fuentes de información, se elaboró un protocolo de codificación, en el que los dos investigadores — de manera independiente- registraron las noticias relacionadas con el objeto de estudio, construyendo así una base de datos con todos los eventos pesquisados (Villalobos, 2019). Utilizando este protocolo, se realizó el proceso de codificación de las noticias de protesta, lo que permitió describir los eventos de protesta estudiantil en términos de las características de sus participantes, las demandas esgrimidas y las tácticas, acciones o repertorios de acción utilizados. Respecto de las características de los participantes, se recolectó información sobre el número de participantes, tipos de participantes (estudiantes secundarios, postsecundarios, docentes, apoderados $u$ otros) $y$ las organizaciones sociales y políticas involucradas (nombres y cantidades). En relación con las demandas, se transcribió literalmente el objetivo declarado de la protesta según los medios de prensa (lo que puede incluir consignas de dirigentes, relatos periodísticos o descripciones de terceros), para luego codificar estos objetivos en categorías no excluyentes (demandas educativas generales, financiamiento, funcionamiento del campo educativo, contenidos, organización, beneficios, problemas de instituciones educativas específicas, incumplimiento de leyes o normas, demandas de otros actores del campo educativo y demandas alusivas a temas no educacionales) Finalmente, respecto de las tácticas, se describieron todas las acciones descritas en los reportes de prensa, las que posteriormente fueron codificadas de acuerdo con la clasificación utilizada por Medel y Somma (2016), que definen un grupo de categorías generales (tácticas pacíficas, artísticas, disruptivas no violentas, violentas y autodestructivas), dentro de las cuales es posible ubicar al conjunto de tácticas de protesta específicas. Cabe agregar que, como criterio general, se buscó codificar variables "duras" por sobre variables "blandas", es decir, incorporar variables en las que la interpretación periodística del evento fuera lo más baja posible (Río, 2008). 


\section{c) Análisis de los datos}

Considerando el carácter descriptivo y cuantitativo del estudio, el análisis de los datos se realizó utilizando distintos indicadores de la serie de datos, en la que el eje de comparabilidad longitudinal es alguna medida temporal de los eventos de protesta; en nuestro caso, el año (Abbott, 2001). Este tipo de análisis permite explorar las trayectorias y ciclos de la movilización, mostrando gráficamente la existencia de cambios temporales en algunas de las variables codificadas, tomando como principal referente de comparación el eje temporal (Olzak, 1989).

\section{Resultados}

La cantidad de eventos de protestas ha sido el indicador más utilizado para estudiar la trayectoria histórica de la conflictividad, tanto en el mundo (Jenkins, Jacob y Agnone, 2003; Koopmans y Rutch, 1999; Ortiz et al., 2013) como en Chile (Aguilera y Álvarez, 2015; Disi, 2018; Somma y Medel, 2017). En el caso estudiado, el total de protestas registradas durante el periodo alcanza un total de 750 eventos, con un promedio anual de 30 eventos $(\mathrm{SD}=12.81 ; \mathrm{SE}=2.6$; IC 95\% [24.7 - 35.3]). Esto implica que, en promedio, cada 10 días se produce un conflicto universitario entre 1990 y 2014.

Ahora bien, ¿cómo es la trayectoria histórica de esta conflictividad? La figura 1 muestra la distribución anual de los eventos de protesta entre 1990 y 2014. Tres aspectos se pueden destacar al respecto. En primer lugar, la trayectoria de la conflictividad universitaria presenta una forma oscilante y cíclica, con momentos de auge y caída constantes durante todo el periodo considerado, y un "piso mínimo" de 13 eventos, lo que es concordante con lo indicado por Tarrow (1997) respecto de los procesos de movilización, aunque es relativamente discrepante con lo indicado por otros investigadores, que han destacado el carácter explosivo de las protestas durante los últimos 
años (Salinas y Fraser, 2012; Bellei, Cabalín y Orellana, 2014). En segundo lugar, la tendencia histórica muestra que, en general, las protestas están presentes durante los 25 años de estudio. De esta forma, y en contra de lo que han planteado algunas investigaciones (Montero, 2018; Ruiz y Boccardo, 2015; Somma, 2012), tanto en los años noventa como en la primera década del siglo XXI los procesos de conflictividad tienen similar presencia. Estadísticamente, esto se visualiza en que la serie de conflictos universitarios tiene una leve asimetría positiva ( $\mathrm{SK}=0,48)$, producida especialmente por el sesgo producido por años como 2000 y 2011, en los que se registran los peaks más altos en el número de eventos, tendiendo a elevar el promedio de los datos. Finalmente, el análisis de la parte superior de la figura 1 muestra que los peaks de los conflictos estudiantiles universitarios no tienen un patrón regular ${ }^{5}$, destacándose a este respecto los años 1992 (38 eventos), 1997 (47 eventos), 2000 (53 eventos), 2001 (43 eventos), 2008 (47 eventos), 2011 (56 eventos) y 2012 (42 eventos). Aunque algunos de estos años han sido destacados por anteriores investigaciones (por ejemplo, la centralidad de las protestas del 1997, relevada por el estudio histórico de Thielemann (2016)), los resultados muestran una imagen distinta a las lecturas más difundidas sobre la trayectoria de la conflictividad universitaria, que han resaltado las protestas del 2011 y de 2006 como puntos de inflexión (es decir, como puntos de cambio inesperados y abruptos) en la trayectoria del movimiento (Aguilera, 106; Aguilera y Álvarez, 2015; Ruiz y Boccardo, 2015; Disi, 2018; Somma, 2012).

\footnotetext{
5 Otra forma de analizar el nivel ondular de una trayectoria de datos es a través del análisis de autocorrelación parcial y total. Los resultados de este ejercicio confirman que la trayectoria de las protestas universitarias permiten concluir que no existen efectos endógenos al interior de la serie de datos, lo que implica que los datos no están sujetos a un patrón constante de comportamiento y que, por lo tanto, la cantidad de eventos de protesta registrados en un año determinado no influye sobre la cantidad de protestas que se registran en los años posteriores (Ortiz, 2018).
} 
Figura 1. Número de eventos de protesta universitaria por año (1990-2014)

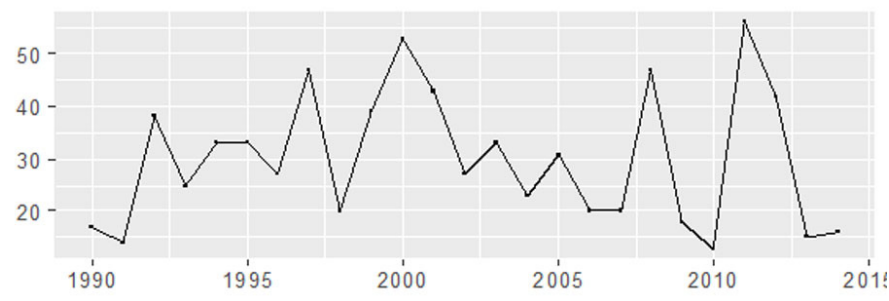

Fuente: elaboración propia.

A pesar de su utilidad, la cantidad de eventos es un indicador que no entrega información sobre todas las particularidades de la evolución de las protestas del movimiento universitario durante el periodo. En consecuencia, para estudiar otras características de estos ciclos se analizan especialmente tres aspectos: las demandas desarrolladas por los actores en los procesos de conflictividad, las tácticas utilizadas durante las protestas y la intensidad de estas acciones.

La figura 2 muestra la frecuencia por año de las demandas universitarias entre 1990 y 2014, organizadas en seis categorías: i) demandas educativas generales (que no especificaban ámbitos $\mathrm{u}$ orientaciones particulares, como por ejemplo "mejorar la calidad educativa"); ii) demandas respecto del financiamiento del sistema universitario; iii) demandas sobre los contenidos $\mathrm{u}$ organización del sistema universitario; iv) demandas sobre beneficios de los estudiantes (por ejemplo, becas); v) demandas internas asociadas a problemas específicos de las instituciones (por ejemplo, mejoramiento de infraestructura); y vi) demandas de solidaridad sobre otros actores educativos. En el conjunto del periodo analizado las demandas respecto del financiamiento del sistema ( $\mathrm{N}=335)$ y las demandas internas $(\mathrm{N}=305)$ son las más frecuentes, representando casi el $70 \%$ del total de los conflictos registrados. En contraste, las demandas sobre contenidos del sistema universitario y las demandas de solidaridad no superan el 10\% del conjunto de los conflictos. 
En términos de su distribución histórica, es posible observar un proceso de heterogenización de las demandas, que es principalmente visible a través de tres aspectos. En primer lugar, las demandas educativas generales tienen una distribución baja durante todo el periodo, excepto en 2011 cuando se observa un peak fuerte $(\mathrm{N}=24)$, lo que se encuentra en línea con estudios que han planteado que lo fundamental de las protestas de ese año fue precisamente que los estudiantes lograron desarrollar demandas generales de transformación del conjunto del sistema educativo (Salinas y Fraser, 2012). En segundo lugar, es posible visualizar procesos de caída de las demandas acerca del financiamiento y el contenido del sistema universitario. En el caso del financiamiento, esta caída se observa especialmente desde 2006, concordante con lo relevado por recientes análisis históricos (Thielemann, 2016), mientras que respecto de los contenidos la caída es evidenciada desde 1998. Ambos aspectos estarían dando cuenta de una disminución del peso de los factores "coyunturales" de la movilización universitaria. Finalmente, las demandas respecto de beneficios estudiantiles, las demandas internas y las protestas de solidaridad tienen un comportamiento cíclico, con altas y bajas durante todo el periodo, aunque llama la atención el peak de demandas internas generado en el año 2000, aspecto poco relevado hasta hoy por la literatura. 
Figura 2. Número de eventos de protesta universitaria según tipo de demanda (1990-2014)
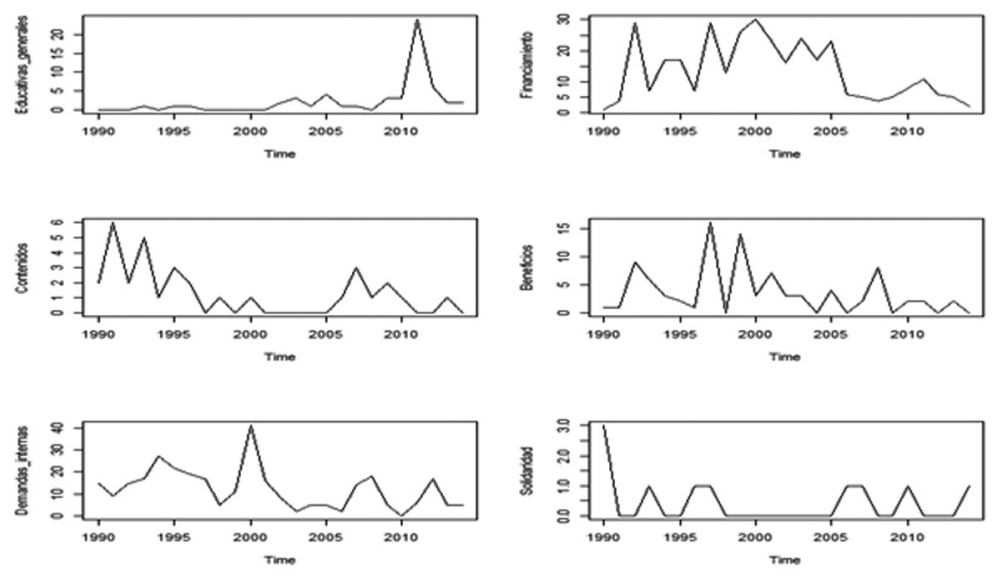

Fuente: elaboración propia.

Nota: los porcentajes no son excluyentes entre sí, ya que un evento de protesta puede contener más de una demanda.

Una segunda característica de las protestas dice relación con las tácticas utilizadas por el movimiento estudiantil universitario. La figura 3 presenta la distribución proporcional (respecto del conjunto de las protestas desarrolladas cada año) de tres repertorios de acción: las marchas, las tomas y las manifestaciones culturales. Mientras las marchas han sido consideradas la táctica o forma de acción contenciosa clásica de los movimientos estudiantiles universitarios (Van Dyke, 2003), las tomas y las manifestaciones culturales o simbólicas, que incluyen performances musicales, teatrales o audiovisuales, que pueden ser realizadas tanto por los manifestantes (por ejemplo, flashmobs o uso de marionetas) o por artistas profesionales (por ejemplo, conciertos $\mathrm{u}$ obras de teatro), han sido caracterizadas como estrategias novedosas desplegadas en los repertorios de movilización estudiantil del país (Ponce y Miranda, 2016; Aguilera, 2017). 
Figura 3. Distribución porcentual de protestas universitarias según táctica: marchas, tomas y manifestaciones simbólicas (1990-2014)

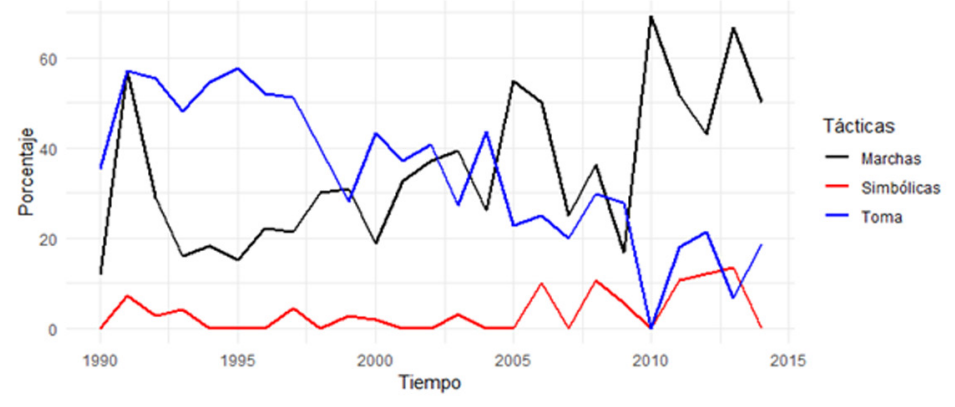

Fuente: elaboración propia.

Nota: los porcentajes no son excluyentes entre sí, ya que un evento de protesta puede contener más de una táctica.

Como se puede observar, las tres tácticas analizadas observan una trayectoria histórica bastante diferente. Por una parte, las tomas muestran una caída constante durante el periodo, pasando de ser la táctica más utilizada a principios de los noventa a no superar más del 20\% durante los últimos años. En contraste, las marchas muestran una tendencia al alza en el conjunto del periodo. Así, mientras entre 1990 y 1999 estas fueron tácticas desarrolladas en el $28 \%$ de las protestas, entre 2000 y 2009 esta cifra se eleva al 37\%, y entre 2010 y 2014 llega al 51\%. Adicionalmente, el análisis temporal muestra que las marchas están especialmente presentes en cuatro ciclos de movilización: en 1991, en 2006, en 2011 y en 2013. De esta forma, y tal como han destacado estudios respecto de acciones contenciosas en otros campos (Somma y Medel, 2017), las demostraciones callejeras constituyen un aspecto central de los procesos de protesta en el Chile actual. Finalmente, las manifestaciones simbólicas o culturales representan un bajo porcentaje del total de protestas durante todo el periodo, aunque es posible visualizar un aumento en la última década, especialmente en los años 2006, 2008, 2011, 2012 y 2013. Estos datos podrían estar indicando un auge de este tipo de tácticas, aunque este aumento no parece tener 
la magnitud ni la centralidad - a lo menos desde la perspectiva cuantitativa - que algunos investigadores han visualizado (Bellei, Cabalín y Orellana, 2014; Paredes, Ortiz y Araya, 2018; Ponce y Miranda, 2016; Salinas y Fraser, 2012).

Finalmente, la última característica a estudiar dice relación con la intensidad de los procesos de conflictividad. Aunque existen muchas formas de medir la intensidad de las protestas, la literatura se ha focalizado especialmente en dos aspectos: su duración y la cantidad y distribución geográfica de los participantes. Mientras el primer indicador da cuenta de la extensión temporal de las protestas, el segundo se refiere a la magnitud de personas involucradas en estos procesos (Fernández, 2014; Disi, 2017).

La figura 4 muestra la trayectoria histórica de la duración promedio (en días) de las protestas registradas anualmente. En el conjunto de las protestas universitarias de la posdictadura, la duración promedio de las protestas es de 4,6 días, existiendo un $38 \%$ de las protestas con un día (o menos) de duración.

Figura 4. Duración promedio de los eventos de protesta universitaria (19902014)

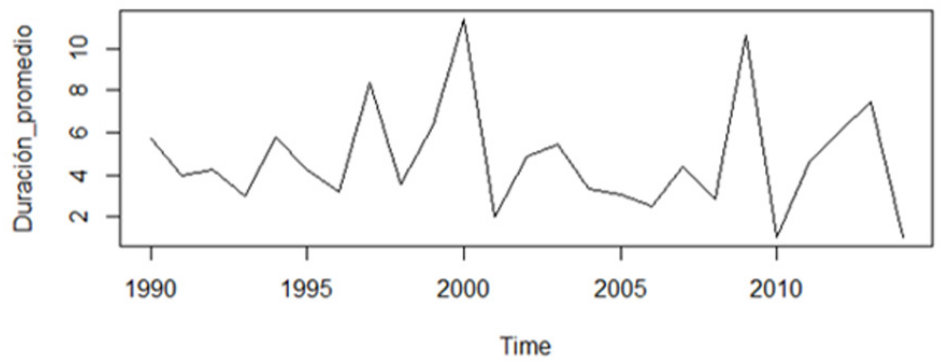

Fuente: elaboración propia.

Resulta interesante destacar dos aspectos de estos resultados. En primer lugar, la duración promedio de las protestas muestra una tendencia oscilante a través del tiempo, con alzas y bajas sucesivas durante todo el periodo, de manera bastante concordante 
con el comportamiento del indicador de cantidad de protestas. Este hallazgo plantea una diferencia a lo señalado por Fernández (2014), que entiende que la extensión de las protestas (aunque en su caso, medida como cantidad de días de protesta al año) ha aumentado constantemente en los últimos años. En segundo término, y cuando se analizan los peaks, se puede observar que 1997, 2000 y 2009 son los años con mayores alzas, con duraciones promedio de 8,$3 ; 11,3 ;$ y 10,6 días respectivamente; lo que podría estar dando cuenta de que en estos puntos de la trayectoria del movimiento se habría producido una masificación de acciones de largo aliento, como ocupaciones o tomas de universidad. Cabe consignar que estos años no han sido especialmente destacados por la literatura existente, a excepción de 1997, referenciado por Thielemann (2016).

Una segunda forma de estudiar la magnitud de la trayectoria histórica de las protestas universitarias es a través del análisis de la cantidad de personas participantes en las protestas o de su distribución geográfica. Al ser un indicador altamente sensible para los medios de prensa (Río, 2008), el número de participantes debe ser analizado e interpretado con precaución. En nuestro caso, el proceso de codificación permitió identificar la cantidad de manifestantes solo en el $85 \%$ de los eventos, los que están representados (en su promedio anual) a través de la figura $5^{6}$.

\footnotetext{
6 Para el cálculo del número de participantes, se incorporó el número exacto reportado por el medio de prensa. En el caso de que distintas noticias (del mismo medio o de distintos medios) reporten números distinto de participantes, se calculó el promedio de participantes según cada reporte. En el caso que una misma noticia reporte distintos números de participantes (por ejemplo, cantidad estimada por los manifestantes vs. cantidad estimada por Carabineros) se calculó este promedio como el dato a reportar por el medio de prensa. Finalmente, en el caso de que el evento de protesta incorporara más de una acción, se sumaron los números de participantes (por ejemplo, cuando se realizan marchas en distintas ciudades) para calcular el número de participantes según ese medio de prensa.
} 
Figura 5. Promedio de participantes por año (1990-2014)

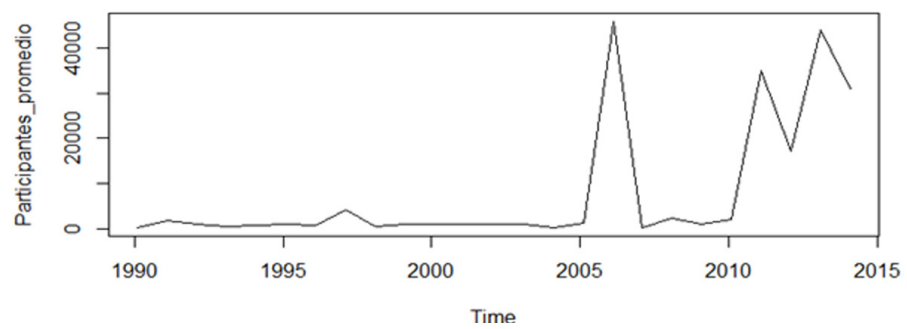

Fuente: elaboración propia.

Como se puede observar, tanto la tendencia histórica como los peaks de este indicador difieren con los presentados anteriormente. En primer término, existe una evidente diferencia respecto de la tendencia cíclica evidenciada en la cantidad de protestas y en la duración promedio de estas, ya que, en este caso, la trayectoria histórica se visualiza con explosivos peaks que no siguen un patrón identificable (Koopmans y Rutch, 1999). En segundo término, la trayectoria muestra una importante diferencia entre la década de los noventa y el periodo posterior. Mientras la cantidad de participantes se mantiene relativamente estable entre 1990 y 2005, fluctuando entre 500 y 5.000 asistentes por evento, desde 2006 se reconoce el inicio de un proceso de escalada de la magnitud de las protestas, con puntos álgidos en los años 2006, 2011 y 2013. En función de lo anterior, se sostiene que 2006 y 2011 constituyen más bien hitos respecto del auge en la intensidad y/o magnitud de las protestas estudiantiles universitarias, antes que sobre el aumento de la frecuencia de los eventos (Aguilera, 2016; Bellei, Cabalín y Orellana, 2014).

Aunque relevante, la cantidad de participantes habla de la magnitud de la protesta, pero no de su extensión territorial. Tal como indica Tarrow (1997), para los movimientos sociales es importante incluir a franjas cada vez más amplias de la población, pero también tener una presencia cada vez más extensa en el territorio. Para ilustrar esta extensión territorial, la figura 6 muestra 
la cantidad de protestas nacionales ${ }^{7}$ universitarias desarrolladas entre 1990 y 2014 en el país. De acuerdo con estos datos, si bien la cantidad de protestas nacionales presenta una cierta ciclicidad, con alzas y bajas durante todo el periodo, lo más llamativo resulta ser la creciente cantidad de protestas nacionales que se registran durante la última década. Así, entre 2004 y 2013 se presentan seis años con 7 o más protestas nacionales ( 9 en 2005, 7 en 2006, 12 en 2008, 17 en 2011, 10 en 2012 y 7 en 2013), lo que podría estar dando cuenta de una consolidación de las orgánicas y de cierta transformación del movimiento universitario (Avendaño, 2014), permitiendo el desarrollo de protestas coordinadas a lo largo del territorio.

Figura 6. Cantidad de protestas nacionales (1990-2014)

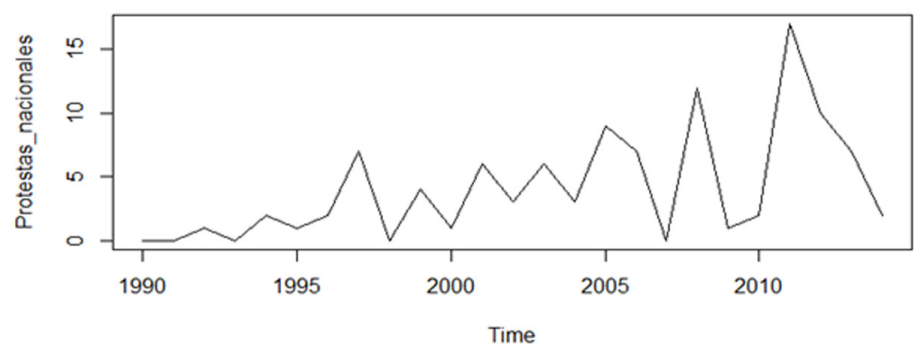

Fuente: elaboración propia.

\section{Conclusiones}

Mediante el uso sistemático de distintas fuentes de prensa, el presente artículo buscó describir la trayectoria de la conflictividad del movimiento estudiantil chileno en la posdictadura, dando cuenta de los principales ciclos, tendencias y características de estas protestas. En este caso, se consideran protestas nacionales aquellas en las que el evento se
produce en, a lo menos, tres regiones no contiguas. 
Vistos en su conjunto, los resultados permiten matizar, complementar o contrarrestar algunas de las tesis esgrimidas por el campo académico respecto de los procesos de conflictividad universitaria en las últimas décadas. Así, por ejemplo, la tesis de que la trayectoria de las protestas universitarias podría caracterizarse por un periodo de desmovilización en la década de los noventa y un aumento de la conflictividad desde los 2000 (Tesis 1), se ve contrarrestada por la existencia de olas cíclicas de alta y baja conflictividad durante todo el periodo y con ciclos de alta densidad de protestas entre 1994 y 1997, poco destacados por la mayoría de las investigaciones. Por otro lado, la idea de que durante los últimos años se habrían producido cambios importantes a nivel de las tácticas y demandas del movimiento (Tesis 2), se ve complementada, puesto que, aunque los datos permiten comprobar una heterogeneización de las demandas y un cambio en la centralidad de ciertas tácticas, no se verifica una tendencia sostenida hacia el aumento de demandas de corte estructural o global, como tampoco una preponderancia de las tácticas performáticas o simbólicas. En cambio, lo que los datos permiten concluir es que más bien la presencia de un periodo de superposición de demandas y tácticas, las que el movimiento universitario despliega según su propia estrategia de movilización y contexto. En tercer lugar, la idea de que la intensidad del conflicto en el campo universitario se ha incrementado en las últimas décadas (Tesis 3) encuentra bastante evidencia a favor en los datos obtenidos en este estudio, fundamentalmente en función del aumento del número de participantes y de la cantidad de protestas nacionales registradas (posiblemente relacionadas con la maduración de su estructura orgánica, liderazgos y construcción de redes durante el periodo), aunque no se detectan cambios relevantes en la duración de los eventos, que tiende a mantenerse estable en todo el periodo observado.

Todos estos resultados permiten construir una imagen más compleja del proceso histórico de movilización estudiantil universitaria en la posdictadura, pudiendo ser entendido tanto a 
partir de sus continuidades como de sus rupturas durante el periodo de estudio. Dicho de otro modo, la conflictividad estudiantil en el Chile posdictatorial no es exclusivamente mantención, ni tampoco sólo novedad, sino más bien ambos aspectos a la vez y mezclados. Como todo movimiento, contiene desafíos y formas de expresión históricas, pero también demandas y tácticas nuevas, surgidas y alimentadas a partir de la historicidad que le imprimen décadas de acción colectiva.

¿Cómo entender este proceso de continuidad y ruptura en los próximos años? ¿Qué aspectos deberían considerarse en próximos estudios para comprender mejor la trayectoria histórica de este movimiento y sus consecuencias? De manera hipotética, y sin pretender exhaustividad, es posible ensayar tres respuestas a estas preguntas. En primer lugar, parece importante considerar de manera más clara la relación entre acción social (protesta) y estructura del campo universitario. Desde 2017 una reforma general de la forma en cómo se organiza y funciona el campo educativo ha sido aprobada, para algunos precisamente como efecto institucional del último ciclo de protestas (Montero, 2018). Más allá de sus causas, lo cierto es que esta reforma ha motivado nuevas discusiones respecto del sentido de los derechos sociales en el espacio universitario, la idea de calidad universitaria o la organización de la educación universitaria pública (Cortés y Villalobos, 2018). Preguntarse por cómo afectarán estos cambios el sentido, lógica y formas de acción de las protestas en el espacio universitario es, por lo mismo, una pregunta central para los próximos estudios sobre esta materia.

Por otra parte, parece necesario considerar más claramente la relación entre lo local y lo global en los procesos de protestas. Tal como algunos autores han destacado ( $\mathrm{Su}, 2015)$, las protestas no son fenómenos cerrados, sino que se propagan transnacionalmente. En esta línea, por ejemplo, Milkman (2017) ha afirmado que las protestas que se han sucedido en distintos países del mundo desde la crisis económica del 2008 se explican por el surgimiento de una nueva generación política mundial (la ge- 
neración millenial $^{8}$ ), pudiendo, por lo mismo, movimientos tan distintos como la "primavera árabe", los "Indignados" en España, "Occupy Wall Street" en Estados Unidos, o y el movimiento estudiantil chileno ser considerados expresiones diversas de un mismo fenómeno global, aspecto relevante pero escasamente estudiado hasta la fecha.

Finalmente, y desde la vereda metodológico-conceptual, la inclusión y difusión de análisis históricos de mediano y largo plazo (como el desarrollado en este artículo) permiten visualizar tendencias y características de los procesos de movilización social que no son del todo nítidos a través de diseños de investigación transversales o que tienen una delimitación temporal más acotada. En este sentido, se espera que este trabajo pueda contribuir a hacer patentes las ventajas y oportunidades que permite la utilización de metodologías de análisis histórico para, de esta manera, complementar y complejizar el análisis del movimiento universitario chileno, promoviendo así una imagen más completa y dinámica de este fenómeno, retomando la máxima de Wright-Mills (1967) de que "la historia es la raíz de los estudios sociales" (p. 143).

Estudiar estos aspectos nos permitirá, desde nuestro punto de vista, aumentar el conocimiento histórico de los procesos de conflictividad de uno de los movimientos más activos del país: el movimiento universitario.

\section{Referencias}

Abbott, A. (2001). Time Matters. On theory and method. Chicago-Londres: University of Chicago Press.

Esta generación estaría compuesta por adultos jóvenes con una especial disponibilidad biográfica hacia la protesta, familiarizados desde pequeños con el uso de nuevas tecnologías de comunicación e información, con más años de estudios y calificación que las generaciones anteriores, pero, a la vez, con grandes frustraciones de expectativas a causa del estrechamiento del mercado laboral a nivel mundial, lo que motivaría en ellos una orientación favorable hacia la protesta. 
Aguilera, O. (2016). Movidas, movilizaciones y movimientos. Cultura política y políticas de las culturales juveniles en el Chile de hoy. Santiago de Chile: RIL.

Aguilera, O. y Álvarez, J. (2015). El ciclo de movilización en Chile 20052012: Fundamentos y proyecciones de una politización. Revista Austral de Ciencias Sociales, 29(1), 5-32.

Akaev, A., Korotayev, A., Issaev, L. y Zinkina, J. (2017). Technological development and protest waves: ¿Arab spring as a trigger of the global phase transition? Technological Forecasting $\mathcal{E}$ Social Change, 116 (2017) 316-321.

Almeida, P. (2007). Defensive mobilization. Popular movement against economic adjustment policies in Latin America. Latin American Perspectives, 154(34), 123-139.

Asún, R., Zúñiga, C., Yañez, L. y Villalobos, C. (2018). ¿Cómo responden las Ciencias Sociales a temas de alta contingencia política?: el caso del movimiento estudiantil chileno. Documento de Trabajo, Facultad de Ciencias Sociales, Universidad de Chile. Santiago de Chile: FACSO.

Avendaño, O. (2014). Fracturas y representación política en el movimiento estudiantil. Última Década, 41(1) 41-68.

Bellei, C., Cabalín, C. y Orellana, V. (2014). The 2011 Chilean student movement against neoliberal educational policies. Studies in Higher Education, 39(3), 426-440.

Brunner, J.J. y Flisfisch, A. (2014). Los intelectuales y las instituciones de la cultura. Santiago de Chile: Ediciones Universidad Diego Portales.

Cabalín, C. (2012). Neoliberal Education and Student Movements in Chile: inequalities and malaise. Policy Futures in Education, 10(2), 219-228.

Calderón, F. (2013). Understanding Social Conflict in Latin America. Buenos Aires: Programa de Naciones Unidas para el Desarrollo.

Canales, M. (2006). Metodologías de investigación social. Introducción a los oficios. Santiago de Chile: LOM.

Cortés, A. y Villalobos, C. (2018). El derecho a la educación en el Chile contemporáneo. Cuatro argumentos para su reconocimiento constitucional. En J. Gajardo y Y . Vásquez (Eds.). Nueva Constitución. Ideas y propuestas para el cambio constitucional (pp. 135-154). Santiago de Chile: Rubicón Editores 
De la Maza, G. (1999). Movimientos Sociales en la Democratización de Chile. En P. Drake e I. Jaksic (Comp.), El Modelo Chileno. Democracia y Desarrollo en los Noventa: Santiago de Chile: LOM.

Della Porta, D. (2008). Eventful protest, global conflicts. Conference of the Nordic Sociological Association. Norway: Aahurs.

Della Porta, D., Kriesi, H. y Rucht, D. (2009). Social movements in a globalizing world. Londres: Palgrave-McMillan.

Demirel-Pegg, T. (2014) From the Streets to the Mountains: The Dynamics of Transition from a Protest Wave to an Insurgency in Kashmir. Mobilization, 19(3), 309-327.

Disi, R. (2017). Still massive? Explaining the size of tertiary student protests in Latin America. Working Paper. Universidad Católica de Temuco.

Disi, R. (2018). Sentenced to Debt: Explaining Student Mobilization in Chile. Latin American Research Review, 53(3), 448-465.

Dodson, K. (2015). Globalization and Protest Expansion. Social Problems, 62(1), 15-39.

Earl, J., Martin, A., McCarthy, J. y Soule, S. (2004). The use of newspaper data in the study of collective action. Annual Review of Sociology, 30, 65-80.

Espinoza, O. y González, L. E. (2014). Los inicios del segundo gobierno de Bachelet bajo la Lupa: Avances, dificultades y desafíos de la "reforma" del Sistema de Educación Superior. En Barómetro de Política y Equidad, Primer Tiempo (pp.78-96). Santiago de Chile: Fundación Equitas-Fundación Friedrich Ebert.

Fernández, J. (2014). La protesta social en Chile (2006-2012). Conflicto social y repertorios de acción en torno a los movimientos estudiantil, mapuche y ambiental. Documento de Trabajo GGIP. Barcelona.

Fuentes, C. (1999). After Pinochet: Civilian Unity, Political Institutions, and the Military in Chile (1990-1998). Working Paper Number 28. Durham, NC: Duke-University of North Carolina Program in Latin American Studies.

Guzmán-Concha, C. (2012). The Students' Rebellion in Chile: Occupy Protestor Classic Social Movement? Social Movement Studies: Journal of Social, Cultural and Political Protest. DOI: 10.1080/14742837.2012.710748. 
Jenkins, J., Jacobs, D. y Agnone, J. (2003). Political opportunities and African-American Protest, 1948-1997. American Journal of Sociology, 109(2), 277-303.

Koopmans, R. (1993). The dynamics of protest waves: West Germany, 1965 to 1989. American Sociological Review, 10(1), 637-658.

Koopmans, R. (2007). Protest in time and space: The evolutions of waves of contention. En D. Snow, S. Soule y H. Kriesi (Eds.), The Blackwell Companion of Social Movements (pp. 19-46). Malden: Blackwell Publishing.

Koopmans, R. y Rucht, D. (1999). Protest Event Analysis. En B. Klandermans y S. Staggenborg (Eds.), Methods of Social Movement Research (pp. 231-259). Minneapolis: University of Minnesota Press.

Medel, R. y Somma, N. (2016). ¿Marchas, ocupaciones o barricadas? Explorando los determinantes de las tácticas de la protesta en Chile. Politica y Gobierno, 23(1): 163-199.

Milkman, R. (2017). A New Political Generation: Millennials and the Post-2008 Wave of Protest. American Sociological Review, 82(1) 1-31.

Montero, V. (2018). Movimientos sociales y consecuencias político-institucionales. Del movimiento universitario 2011 a la reforma en educación superior. Persona y Sociedad, 32(2), 46-68.

Olzak, S. (1989). Analysis of events in the study of collective action. Annual Review of Sociology, 15, 119-141.

Olzak, S. y West, E. (1991). Conflict and the rise and fall of ethnic newspapers. American Sociological Review, 56(4), 458-474.

Ortiz, C, (2018). Estructura social y movimiento estudiantil en el Chile postdictadura (1990-2014). Tesis para optar al grado de Magíster en Sociología. Facultad de Ciencias Sociales, Universidad de Chile. Santiago de Chile.

Ortiz, D., Myers, D., Walls, E. y Diaz, M-E. (2005). Where do we stand with newspaper data? Mobilization, 10(3), 397-419.

Paredes, J. P., Ortiz, N. y Araya, C. (2018). Conflicto social y subjetivación política: performance, militancias y memoria en la movilización estudiantil post 2011. Persona y Sociedad, 32(2), 122-149.

Petitat, A. (1987). School and the production of society. British Journal of Sociology of Education, 8(4), 379-390. 
Ponce, C. y Miranda, N. (2016). Redes de confianza online y flash mobs: movilizados por la educación. Observatorio (OBS). Journal, Media, Internet and Social Movements in the context of asymmetries, 161-175.

Reca, I. (1970). El movimiento estudiantil y el proceso de reforma de la Universidad de Chile. Revista Mexicana de Sociología, 32(4), 893-947.

Río, M. (2008). Usos y abusos de la prensa como fuente de datos sobre acciones colectivas. Revista de Metodología de Ciencias Sociales, 16(1), 59-84.

Ruiz, C. y Boccardo, G. (2015). Los chilenos bajo el neoliberalismo. Clases y conflictos sociales. Santiago de Chile: Ediciones El Buen Aire S.A.

Salinas, D. y Fraser, P. (2012). Educational opportunity and contentious politics: The 2011 Chilean student movement. Berkeley Review of Education, 3(1), 17-47.

Salinas, S. (2016). Conflictos y Nuevos Movimientos Sociales. Santiago de Chile: RIL Editores.

Seferiades, S. (2010). 1968 as an epistemological catalyst: Contentious Politics and Antinomies in the Study of Social Movements. Historein, 9, 101-115.

Scherman, A., Arriagada, A. y Valenzuela, S. (2015). Student and Environmental Protests in Chile: The Role of Social Media. Politics, 35(2), 151-171.

Snow, D., Soule, S. y Kriesi, H. (2007). The Blackwell Companion to social movements. Malden: Blackwell Publishing.

Somma, N. (2012). The Chilean student movement of 2011-2012: Challenging the marketization of education. Interface. Journal for and about Social Movements, 4(2), 296-309.

Somma, N. y Medel, R. (2017). Shifting relationship between social movements and institutional politics. En S. Donoso y M. von Bülow (Eds.), Social Movements in Chile Organization, Trajectories, and Political Consequences (pp. 23-62). Nueva York: Palgrave-McMillan.

Su, Y-P. (2015). Anti-Government protest in democracies. Comparative Politics, 48(1), 149-167.

Tarrow, S. (1997). El poder en movimiento. Movimientos sociales, acción colectiva y política. Madrid: Alianza. 
Thielemann, L. (2016). La anomalía social de la transición. Movimiento estudiantil e izquierda universitaria en el Chile de los noventa (19872000). Santiago de Chile: Tiempo Robado.

Tilly, C. y Wood, L. (2013). Los movimientos sociales. 1768-2008. Desde sus orígenes a Facebook. Barcelona: Crítica.

Treviño, E., Villalobos, C., Béjares, C. y Naranjo, E. (2018). Forms of youth political participation and educational system. The role of the school for 8th grade in Chile. Young, 27(3), 1-25.

Touraine, A. (1970). El movimiento de Mayo o el comunismo utópico. Buenos Aires: Signos.

Van Dyke, N. (2003). Crossing movement boundaries: factors that facilitate coalition protest by American College Student, 19301990. Social Problems, 50(2), 226-250.

Vasconi, T. (1968). Educación y cambio social. Santiago de Chile: CESO.

Villalobos, C. (2015). Los conflictos sociales en las sociedades contemporáneas. Apuntes conceptuales y metodológicos desde el realismo modesto. Cuadernos de Trabajo Social, 12(1), 79-93.

Villalobos, C. (2019). Los conflictos sociales en el campo educativo en el Chile posdictadura. Análisis de su evolución, principales características y factores relacionados. Tesis para optar al grado de Doctor en Ciencias Sociales. Facultad de Ciencias Sociales, Universidad de Chile. Santiago de Chile.

Von Bülow, M. y Donoso, S. (2017). Introduction. Social movements in contemporary Chile. En S. Donoso y M. von Bülow (Eds.). Social Movements in Chile. Organization, trajectories and political consequences (pp. 3-28). New York: Palgrave-Macmillan.

Wright-Mills, C. (1961). La imaginación sociológica. México D.F.: Fondo de Cultura Económica. 